\title{
Recurrent Toxic Epidermal Necrolysis Syndrome: A Report of Two Cases
}

\author{
Claudia Reynoso-von Drateln ${ }^{1}$ - Noemí Gómez-Hernández ${ }^{1}$. \\ Norma Rodríguez-Martínez ${ }^{1}$ - Carlos Torres-Lozano ${ }^{1}$
}

Published online: 13 September 2016

(c) The Author(s) 2016. This article is published with open access at Springerlink.com

\begin{abstract}
An 81-year-old male and a 47-year-old female experienced recurrent severe bullous dermatosis secondary to an intake of drugs and alternative medicines indicated for arthralgias. The first patient had previously presented with Stevens-Johnson/toxic epidermal necrolysis (TEN) overlap syndrome in 2007 secondary to ingestion of trimethoprim/sulfamethoxazole indicated for a urinary tract infection; 6 years later, he presented with the same syndrome 2 days after ingestion of oral naproxen tablets $250 \mathrm{mg}$ twice daily. The second patient had presented 5 years previously with TEN after receiving trimethoprim/sulfamethoxazole. In 2014, she presented with arthralgias and received a xenobiotic oral called 'miracle pills' (dosage is unknown); 3 weeks later, she again experienced TEN. Both patients were treated with intravenous immunoglobulin $400 \mathrm{mg} / \mathrm{kg} / \mathrm{day}$; duration of treatment was 5 days for the first patient and 3 days for the second. However, the male patient died from severe sepsis; the female patient experienced a favorable outcome. There are many risk factors for the development of cutaneous adverse drug reactions; a history of allergic reactions is one important risk factor, and both patients had it. This article reviews the scientific literature on this topic and analyzes the possible causes, including infectious processes, immunological defects, and immunogenetic factors.
\end{abstract}

Claudia Reynoso-von Drateln

vondrateln02@yahoo.com.mx

1 High Specialty Medical Unit, National Medical Center, Specialities Hospital, Belisario Domínguez núm. 1000. Col. Independencia Oriente, CP 44340 Guadalajara, Jalisco, México

\section{Key Points}

Stevens-Johnson syndrome and toxic epidermal necrolysis can be recurrent for different drugs.

History of adverse drug reactions is an important risk factor for the development of hypersensibility to medication.

Alternative medicine can produce cutaneous severity reactions.

\section{Introduction}

Stevens-Johnson syndrome (SJS) and toxic epidermal necrolysis (TEN) or Brocq-Lyell syndrome are dermatoses triggered principally by drugs [1]. Cases reported as recurrent have been attributed to infectious or environmental factors [2]. However, most cases in the pediatric population are not related to recurrences secondary to drug intake. Demonstrating the causal medication with certainty is complex [3].

We present two cases. The first was a man with TEN secondary to naproxen intake in whom sulfonamides had previously triggered the same syndrome. The second was a woman with a history of SJS after ingesting sulfonamides and who presented, in her most recent hospitalization, with TEN triggered by alternative medicines.

\section{Clinical Cases}

\section{Patient 1}

The patient was male, 81 years old, and had a 30-year history of alcoholism. He presented with overlapping SJS/ 
TEN syndrome in 2007 secondary to ingestion of trimethoprim/sulfamethoxazole indicated for a urinary tract infection. In that instance, he required hospitalization for 1 week and was discharged to his home. This time, 6 years later, he presented with the same syndrome 2 days after ingestion of oral naproxen tablets $250 \mathrm{mg}$ twice a day indicated as an analgesic.

He was admitted to our hospital unit with dermatosis affecting $20 \%$ of his body surface manifesting as flaccid blisters on an erythematous base, a positive Nikolsky sign, conjunctivitis, and ulcers in the oral mucosa (Fig. 1). He had a Score of Toxic Epidermal Necrosis (SCORTEN) score of 4 points. He presented with signs and symptoms of sepsis, hyperglycemia, and elevated ureic nitrogen. He was treated with intravenous immunoglobulin $(400 \mathrm{mg} / \mathrm{kg} /$ day completing a 5-day course); however, on hospital day 15 , he developed multiple organ failure and unfortunately died.

\section{Patient 2}

Patient 2 was a 47-year-old female with a history of oophorectomy at the age of 17 years for left ovarian teratoma, rheumatoid arthritis, lupus erythematosus, fibromyalgia, and dry eye diagnosed 8 years previously. She had a history of TEN 5 years ago triggered by trimethoprim/sulfamethoxazole without apparent sequelae. She received methotrexate $7.5 \mathrm{mg} /$ week, prednisone $5 \mathrm{mg} /$ day, chloroquine $200 \mathrm{mg} / \mathrm{day}$, and sulfasalazine $500 \mathrm{mg} /$ day before hospitalization.

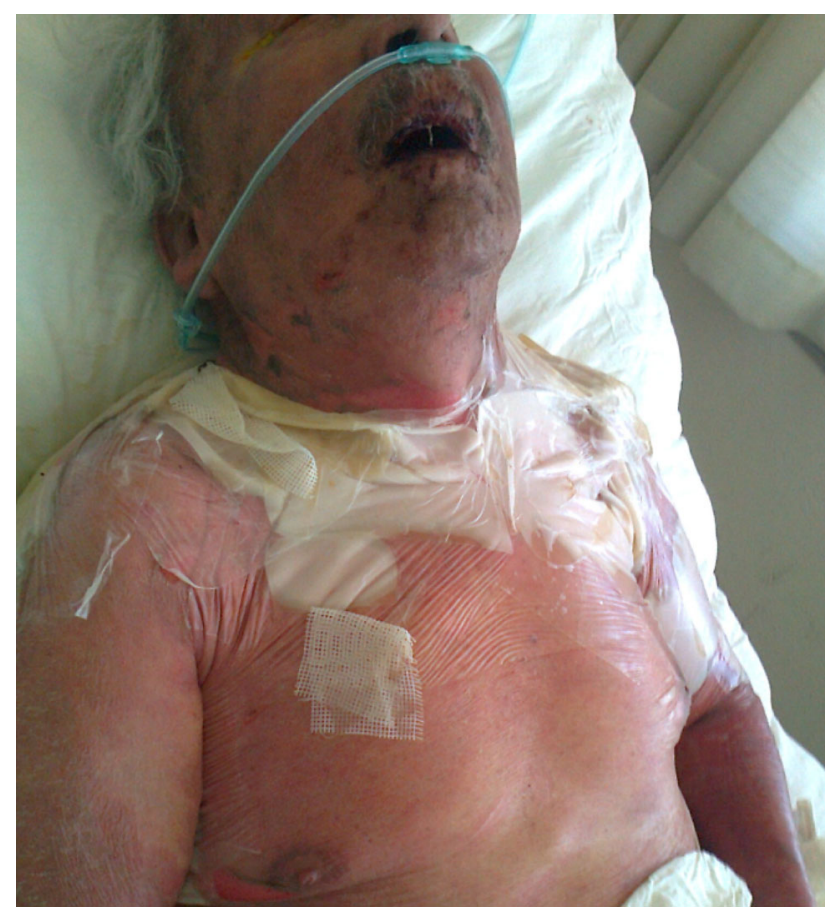

Fig. 1 Toxic epidermal necrolysis
Her symptoms began 3 weeks after she had taken a xenobiotic called 'miracle pills' indicated for arthralgia (this type of medication is used in alternative therapy). Symptoms started with pruritus and erythema in the lumbar region that extended to $75 \%$ of the body surface; blisters appeared later. We do not know what dose of the medication she received, and we suspended treatment as soon as we diagnosed the adverse drug reaction.

Nikolsky's sign was detected on admission, along with friable skin and large areas of denudation with involvement of the ocular and buccal mucosa. A diagnosis of TEN was made. Treatment was started with intravenous methylprednisolone $80 \mathrm{mg} /$ day for 10 days and intravenous immunoglobulin $400 \mathrm{mg} / \mathrm{kg} / \mathrm{day}$ for 3 days. Surgical cleanings were performed in the burn unit. She entered remission of mucocutaneous lesions within 7 days and was discharged to her home 15 days after admission to hospital.

\section{Discussion}

SJS and TEN are rare diseases that occur in approximately two cases per million inhabitants per year; however, they have a considerable effect on the public health of any country because of their high mortality rate, which ranges between 20 and $25 \%$ [4]. Recurrent disease is even rarer and has been reported more frequently in the pediatric population.

Various articles in the scientific literature have reported recurrent disease for SJS and TEN (Table 1). Brown et al. [3] reported a case of SJS with three episodes of recurrence in an adult, apparently secondary to a monomeric non-ionic contrast medium called lopromide.

Casselman et al. [5] reported the case of an 11-year-old girl with recurrent SJS, with seven episodes over a period of 8 years associated with Mycoplasma or a viral infection secondary to dysfunction of the innate immune system, particularly natural killer (NK) cells.

Finkelstein et al. [6] reported ten children with recurrent SJS from a total of 55 study subjects (18\% of the total pediatric population studied).

The etiopathogenic mechanisms that could explain why a patient presents with this skin disease on more than one occasion are unknown. However, it is possible that medications with very similar stereochemical structures administered to the same patient may trigger recurrent SJS or TEN. This may occur because cross-reactivity triggers mechanisms of hypersensitivity to the causal antigen. On the other hand, we consider the possibility that recurrent infections in patients with immune dysfunction or possible primary immunodeficiency and immune-inflammatory responses against microbial agents, such as the Herpes simplex type virus and bacteria such as Mycoplasma 
Table 1 Recurrent StevensJohnson syndrome/toxic epidermal necrolysis case reports

\begin{tabular}{llll}
\hline Study & Patients $(N)$ & Age of presentation (years) & Association \\
\hline Daubeney and Scopes [2] & 1 & 11,12 & Mycoplasma pneumoniae \\
Hartel et al. [11] & 1 & 5,16 & Winter \\
Brown et al. [3] & 1 & 37 & Lopromide contrast \\
Casselman et al. [5] & 1 & 11 & Natural killer cells dysfunction \\
Campagna et al. [12] & 1 & 12 & M. pneumoniae \\
Finkelstein et al. [6] & 10 & $1.8 / 13 / 15$ & 1. M. pneumoniae \\
& & $2.10 / 13$ & 2. M. pneumoniae \\
& $3.14 / 17$ & 3. Herpes simplex \\
& $4.15 / 21$ & 4. Carbamazepine/zonisamide \\
& & $5.10 .6 / 10.8$ & 5. M. pneumoniae/H. simplex \\
& $6.14 / 15$ & 6. Phenytoin/lamotrigine \\
& $7.11 / 12.7$ & 7. M. pneumoniae \\
& $8.11 / 12.5$ & 8. M. pneumoniae \\
& $9.14 .3 / 14.7$ & 9. M. pneumoniae/amoxicillin \\
& $10.14 .5 / 14.8$ & 10. Lamotrigine/unknown \\
\hline
\end{tabular}

pneumoniae, may explain the phenomenon of attack on the stratum corneum cells within the skin [5]. The other possibility concerns the use of medications that act as superantigens, non-covalently bind to T-lymphocyte receptors and/or the histocompatibility molecules, and trigger the immune system per se without prior sensitization, implied by the recently proposed $\mathrm{p}-\mathrm{i}$ concept (pharmacological interaction with immune receptors) [7]. Another etiopathogenic possibility that explains the recurrence of this type of blistering disease is the immunogenetic variability that all human beings have, such as Asian patients who carry the haplotype HLA-B*1502, which is associated with a $100 \%$ risk for presenting with SJS secondary to carbamazepine [8]. Finally, it is important to mention the hepatic detoxification system, including the cytochrome P450 (CYP) enzyme complex. This may be responsible for producing certain drug metabolites that influence the recurrence of SJS and/or TEN, as is the case with the hepatotoxic product $N$-acetyl- $p$-benzoquinoneimine from acetaminophen [9].

Our hypothesis regarding the recurrence of the above syndromes in our patients is as follows. Patient 1 ingested naproxen prior to the second event. This drug is metabolized by CYP2C, and it is well known that the activity of the CYP complex decreases as humans get older. It has been mentioned that one factor influencing the great diversity of responses to medications is changes in the immunogenetics of the CYP complex [10]. On the other hand, people aged $>60$ years present a higher risk of adverse drug reactions because they often ingest more than one medication, have relatively more fat for storage, their kidneys are less able to excrete drugs in the urine, and their liver less effectively metabolizes many medications [11].
Patient 2 had a history of hypersensitivity to trimethoprim/sulfamethoxazole and presented with the skin reaction following the ingestion of an alternative therapy. It is possible that the xenobiotic contained chemical products similar to the previous antibiotic and the immune system triggered an inflammatory response against stratum corneum cells as a result of a cross-reaction; however, we were unable to confirm this.

This case report represents the first Mexican experience and an addition to the few studies we could find, as shown in Table 1. The prevention of recurrent attacks depends on identifying the cause of the first immune-inflammatory attack on the dermoepidermal cellular target. It is important to note that any patient who has had a previous hypersensitivity reaction to drugs is at high risk for recurrence of these syndromes and therefore represents an immune etiopathogenic challenge; our obligation will be to avoid subsequent clinical episodes. The study of immunomolecular and immunogenetic markers to determine the risk of adverse drug reactions constitutes a line of research that has not yet been consolidated and that, once implemented, could reduce morbidity and mortality in our patients.

\section{Compliance with ethical standards}

Funding No financial support was received for the conduct of this study or preparation of this manuscript.

Conflict of interest Claudia Reynoso-von Drateln, Noemí GómezHernández, Norma Rodríguez-Martínez, Carlos Torres-Lozano have no conflicts of interest.

Consent statement We obtained written informed consent from the patients for publication of these case reports and any accompanying images. In the first case, the written informed consent was obtained from the patient's next of kin (daughter) because he had died. 
Open Access This article is distributed under the terms of the Creative Commons Attribution-NonCommercial 4.0 International License (http://creativecommons.org/licenses/by-nc/4.0/), which permits any noncommercial use, distribution, and reproduction in any medium, provided you give appropriate credit to the original author(s) and the source, provide a link to the Creative Commons license, and indicate if changes were made.

\section{References}

1. García Duval I, Roujeau JC, Cruces Prado MJ. Toxic epidermal necrolysis and Stevens-Johnson syndrome: classification and current therapeutics. Actas Dermosifiliogr. 2000;91:541-51.

2. Daubeney PEF, Scopes JW. Recurrent Stevens-Johnson syndrome. J R Soc Med. 1991;84:168.

3. Brown M, Yowler C, Brandt C. Recurrent toxic epidermal necrolysis secondary to Iopromide contrast. J Burn Care Res. 2013;34:e53-6.

4. Kim HI, Kim SW, Park GY, Kwon EG, Kim HH, Jeong JY, Chang HH, Lee JM, Kim NS. Korean J Intern Med. 2012;27:203-10.

5. Casselman J, Venglarcik J, Bludorn M 3rd, Chernin L, Swender D, Tcheurekjian H, Hostoffer R. Association of natural killer cell dysfunction and Stevens-Johnson syndrome in a pediatric patient. Allergy Rhinol. 2013;4(1):e25-6.

6. Finkelstein Y, Soon GS, Acuna P, George M, Pope E, Ito S, Shear NH, Koren G, Shannon MW, Garcia-Bournissen F. Recurrence and outcomes of Stevens-Johnson syndrome and toxic epidermal necrolysis in children. Pediatrics. 2011;128:723-8.

7. Pichler WJ. The $\mathrm{p}-\mathrm{i}$ concept: Pharmacological interaction of drugs with immune receptors. World Allergy Organ J. 2008;1(6):96-102.

8. Lim SK, Kwan P, Tin Tan C. Association of the HLA-B*1502 allele and carbamazepine-induced severe adverse cutaneous drug reactions among Asians, a review. Neurol Asia. 2008;13:15-21.

9. Orellana BM, Guajardo TV. Cytochrome P450 activity and its alteration in various pathologies. Rev Méd Chile. 2004;132:85-94.

10. Chelo-Nuñez H. Cytochrome P450 and its role in drug-induced hepatotoxicity. Enf Ap Dig. 1999;2:34-7.

11. Hartel C, Entenmann A, Wygold T. Recurrent Stevens Johnson syndrome in winter. Pediatr Dermatol. 2004;21(3):282.

12. Campagna C, Tassinari D, Bernardij NF. Mycoplasma pneumoniae induced recurrent Stevens-Johnson syndrome in a children: a case report. Pediatr Dermatol. 2013;3(5):624-6. 